\title{
Coupling Monte Carlo simulations with thermal analysis for correcting microdosimetric spectra from a novel micro- calorimeter
}

Dol:

10.1016/j.radphyschem.2017.02.055

\section{Document Version}

Accepted author manuscript

Link to publication record in Manchester Research Explorer

Citation for published version (APA):

Fathi, K., Galer, S., Kirkby, K., Palmans, H., \& Nisbet, A. (2017). Coupling Monte Carlo simulations with thermal analysis for correcting microdosimetric spectra from a novel micro-calorimeter. Radiation Physics and Chemistry. https://doi.org/10.1016/j.radphyschem.2017.02.055

\section{Published in:}

Radiation Physics and Chemistry

\section{Citing this paper}

Please note that where the full-text provided on Manchester Research Explorer is the Author Accepted Manuscript or Proof version this may differ from the final Published version. If citing, it is advised that you check and use the publisher's definitive version.

\section{General rights}

Copyright and moral rights for the publications made accessible in the Research Explorer are retained by the authors and/or other copyright owners and it is a condition of accessing publications that users recognise and abide by the legal requirements associated with these rights.

\section{Takedown policy}

If you believe that this document breaches copyright please refer to the University of Manchester's Takedown Procedures [http://man.ac.uk/04Y6Bo] or contact uml.scholarlycommunications@manchester.ac.uk providing relevant details, so we can investigate your claim.

\section{OPEN ACCESS}




\section{Author's Accepted Manuscript}

Coupling Monte Carlo simulations with thermal analysis for correcting microdosimetric spectra from a novel micro-calorimeter

K. Fathi, S. Galer, K.J. Kirkby, H. Palmans, A. Nisbet

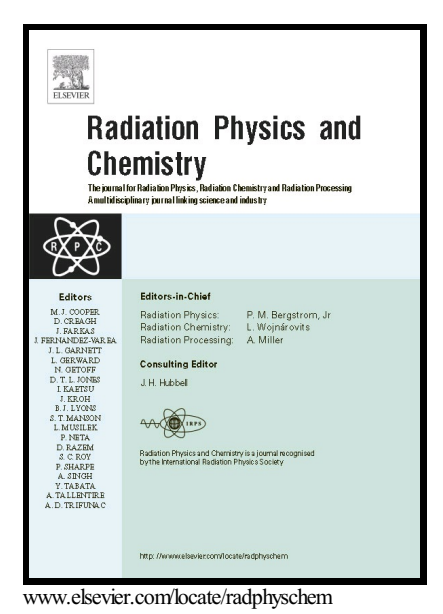

PII: $\quad$ S0969-806X(17)30253-0

DOI: $\quad$ http://dx.doi.org/10.1016/j.radphyschem.2017.02.055

Reference: RPC7457

To appear in: Radiation Physics and Chemistry

Received date: 27 September 2016

Revised date: 22 February 2017

Accepted date: 24 February 2017

Cite this article as: K. Fathi, S. Galer, K.J. Kirkby, H. Palmans and A. Nisbet Coupling Monte Carlo simulations with thermal analysis for correctin: microdosimetric spectra from a novel micro-calorimeter, Radiation Physics an Chemistry, http://dx.doi.org/10.1016/j.radphyschem.2017.02.055

This is a PDF file of an unedited manuscript that has been accepted fo publication. As a service to our customers we are providing this early version o the manuscript. The manuscript will undergo copyediting, typesetting, an review of the resulting galley proof before it is published in its final citable form Please note that during the production process errors may be discovered whic could affect the content, and all legal disclaimers that apply to the journal pertain 


\title{
Coupling Monte Carlo simulations with thermal analysis for correcting
}

\section{microdosimetric spectra from a novel micro- calorimeter}

\author{
K.Fathi ${ }^{\mathbf{1}, \mathbf{7}}$, S. Galer ${ }^{2}$, K. J. Kirkby ${ }^{3,4}$, H. Palmans ${ }^{2,5}$ and A. Nisbet ${ }^{1,6}$ \\ ${ }^{1}$ Department of Physics, University of Surrey, Guildford, UK \\ ${ }^{2}$ Acoustic and lonising Radiation, National Physical Laboratory, Teddington, UK \\ ${ }^{3}$ Molecular and Clinical Cancer Science, University of Manchester, Manchester, UK \\ ${ }^{4}$ The Christie NHS Foundation Trust, Manchester, UK \\ ${ }^{5}$ EBG MedAustron GmbH, A-2700 Wiener Neustadt, Austria \\ ${ }^{6}$ Medical Physics department, Royal Surrey County Hospital, Guildford, UK \\ ${ }^{7}$ Cambridge University Hospital, Cambridge, UK
}

Abstract:

The high uncertainty in the Relative Biological Effectiveness (RBE) values of particle therapy beam, which are used in combination with the quantity absorbed dose in radiotherapy, together with the increase in the number of particle therapy centres worldwide necessitate a better understating of the biological effect of such modalities.

The present novel study is part of performance testing and development of a micro-calorimeter based on Superconducting QUantum Interference Devices (SQUIDs). Unlike other microdosimetric detectors that are used for investigating the energy distribution, this detector provides a direct measurement of energy deposition at the micrometer scale, that can be used to improve our understanding of biological effects in particle therapy application, radiation protection and environmental dosimetry. Temperature rises of less than $1 \mu \mathrm{K}$ are detectable and when combined with the low specific heat capacity of the absorber at cryogenic temperature, extremely high energy deposition sensitivity of approximately $0.4 \mathrm{eV}$ can be achieved.

The detector consists of 3 layers: tissue equivalent (TE) absorber, superconducting (SC) absorber and silicon substrate. Ideally all energy would be absorbed in the TE absorber and heat rise in the superconducting layer would arise due to heat conduction from the TE layer. However, in practice direct particle absorption occurs in all 3 layers and must be corrected for. 


\section{ACCEPTED MANUSCRIPT}

To investigate the thermal behavior within the detector, and quantify any possible correction, particle tracks were simulated employing Geant4 (v9.6) Monte Carlo simulations. The track information was then passed to the COMSOL Multiphysics (Finite Element Method) software. The 3D heat transfer within each layer was then evaluated in a time-dependent model. For a statistically reliable outcome, the simulations had to be repeated for a large number of particles. An automated system has been developed that couples Geant4 Monte Carlo output to COMSOL for determining the expected distribution of proton tracks and their thermal contribution within the detector.

The correction factor for a $3.8 \mathrm{MeV}$ proton pencil beam was determined and applied to the expected spectra. The corrected microdosimetric spectra was shown to have a good agreement with the ideal spectra.

Keywords: Particle therapy; Calorimetry; Microdosimetry; Monte Carlo Simulations; Thermal analysis

\section{1- Introduction}

Particle therapy has the advantage of more localised deposition of dose compared to photons and consequently, less complication of the adjacent healthy tissues.

Since the first use of high energy proton beams for treating cancer in the mid-1950s there has been a rise in the number of proton and ion therapy centres and is projected to increase further. Latest statistics published by the Particle Therapy Co-Operative Group (PTCOG) in 2016, show that there are 68 centres in operation worldwide, 31 under construction and 17 in planning stages (Particle Therapy Co-Operative Group, 2016)

The effective dose in particle therapy is determined by multiplying the physical dose by a Relative Biological Effectiveness (RBE) factor (Paganetti et al., 2002). Accurate determination of the RBE value is necessary for utilising particle therapy to its full potential. There have been a number of in-vivo and in-vitro studies suggesting different values for the RBE for equivalent beams (Daşu and TomaDaşu, 2008; Jones and Dale, 2000; Matsuura et al., 2010; Paganetti et al., 2002; Tilly et al., 2007).

The biological (and other structural) effects of radiation are not only influenced by the average energy deposited per unit mass (i.e. absorbed dose) but also by the number of interactions in a volume of interest (for example nucleus, cell, tissue), their magnitude (amount of energy transferred) and their spatial distribution. Microdosimetry, which defines concepts and quantities to specify the energy concentration in microdosimetric regions, can be employed to improve our understanding of radiation effects at the cellular scale (Kellerer, 1985, 1984; Microdosimetry ICRU Report 36, 1983). The determination of the RBE using microdosimetric spectra for intercomparison of different clinical radiotherapy beams has been demonstrated by Brenner and Zaider (1998). 


\section{ACCEPTED MANUSCRIPT}

The experimental methods in microdosimetry often measure ionisations in gases or semiconductors that are not necessarily representative of the energy depositions and ionisations in tissue. Tissue Equivalent Proportional Counters (TEPCs) are the most commonly used microdosimetry devices and have been attractive due to their ability to amplify the ionisation from a single particle passage (event) into a detectable signal. However, their large sensitive volume (usually centimetre scale) and low energy resolution has led to design and development of many other detectors which work on similar principles. These include the miniaturized TEPCs (mini-TEPCS) designed at the Istituto Nazionale di Fisica Nucleare-Laboratori Nazionali di Legnaro (INFN-LNL) with a sensitive volume on the millimetre scale instead of the centimetre scale (De Nardo et al., 2004; Moro et al., 2006). The mini-TEPCs still suffer from large sensitive volumes relative to the scale desired in microdosimetry measurements (micrometre). In addition, only the ionisations, disregarding excitations and local heating, are considered as a representation of the energy deposited in the counter.

The design concept of a novel Superconductive Quantum Interference Device (SQUID) based microcalorimeter for the determination of microdosimeteric spectra was previously presented by Galer et al. (2011). A typical image of the device is shown in figure (1). To convert the signal obtained from the micro-calorimeter accurately to the microdosimetric spectrum, correction factors are required, which are the focus of this work. During irradiation, energy is deposited along the path of the particle passing through a dual absorber located within the SQUID loop. This dual absorber consists of a superconducting ( $\mathrm{SC}$ ) and a tissue-equivalent (TE) absorber as shown in figure (1).

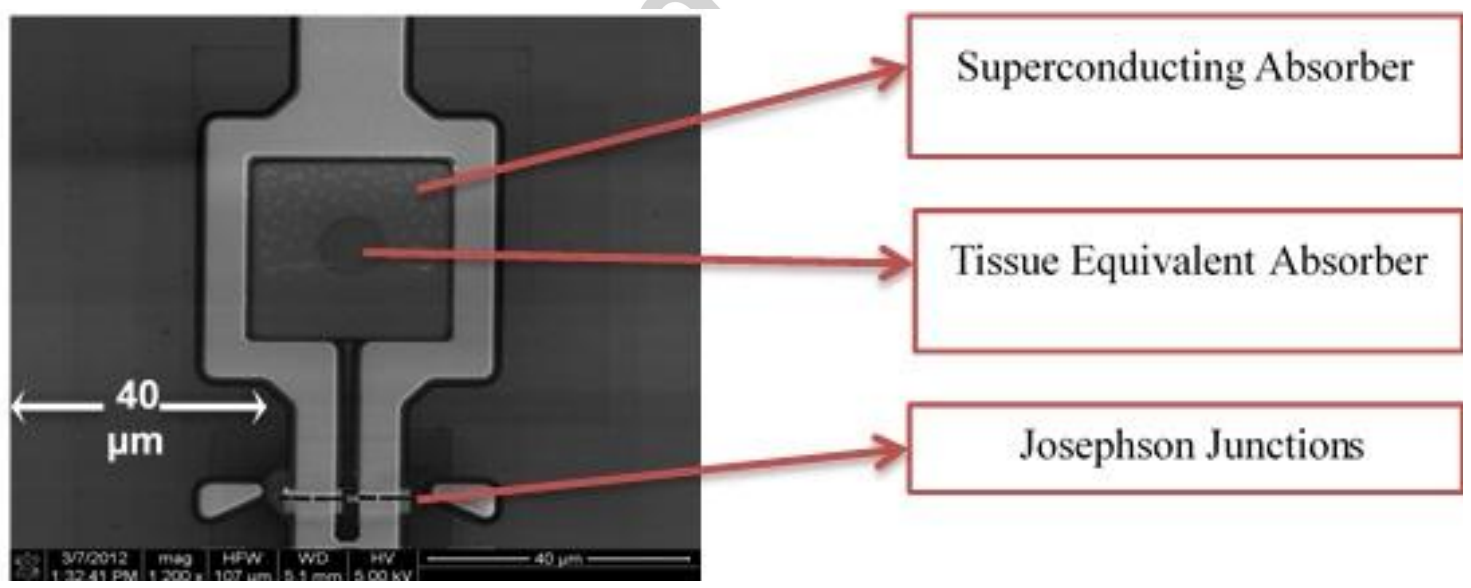

Figure 1: Scanning electron microscope image of the micro-calorimeter.

The energy deposited and its distribution in the TE absorber provides the microdosimetric information directly relevant for the comparison of different treatment modalities for radiotherapy. The quantity of interest is the energy deposited in the TE absorber by a traversing particle which is then converted to lineal energy. In an ideal situation, the traversing particle will deposit energy in the TE absorber alone leading to a temperature rise within its volume. The temperature is then 


\section{ACCEPTED MANUSCRIPT}

conducted from the TE absorber to the SC absorber. This will cause a change in the effective area of the SC absorber which will be detected as a voltage change to the SQUID response to an applied magnetic field. The amount of temperature rise causing the voltage change can be derived as shown by Hao et al. (2003) in their work on Inductive superconducting transition-edge detectors for singlephoton or macro-molecule detection. The SQUID's response to temperature changes in the SC absorber is given by equation (1):

$$
\frac{d V}{d T} \approx \frac{6 \pi R_{d y n} i_{c} \mu_{0}^{2} a}{L^{2}} \frac{\lambda(0) T^{3}}{\left(1-\left(\frac{T}{T_{c}}\right)^{4}\right)^{2 / 3}}
$$

Where

$V$ is the voltage

$T$ is the temperature of the superconducting absorber

$R_{d y n}$ is the dynamic resistance of the SQUID at the bias point

$I_{c}$ is the critical current of the SQUID

$T_{c}$ is the transition temperature of the superconducting absorber

$\lambda(0)$ is the penetration depth at $\mathrm{T}=0 \mathrm{~K}$

$a$ is the radius of the SQUID loop

$L$ is the inductance of the SQUID loop

$\mu_{0}$ is permeability of free space

However, the ideal situation described above cannot be realized since not all heat from the TE absorber will flow to the SC absorber. If the particle is not stopped in the TE absorber, heat will also be generated in the $\mathrm{SC}$ absorber and heat from the $\mathrm{SC}$ absorber can flow to the silicon substrate.

The method of determining those corrections for a 3.8 MeV proton pencil beam are described here. Furthermore, the thermal relaxation behaviour of the novel micro-calorimeter is investigated. A model was created employing Monte Carlo (MC) simulations to determine the energy deposition in the micro-calorimeter. The outcome of the MC simulations were used in a heat transfer model to investigate the thermal response of the micro-calorimeter caused by individual incident particles. 


\section{ACCEPTED MANUSCRIPT}

The process of coupling the two models was automated enabling the analysis of a large number of incident particles at various energies.

\section{2- Method}

\section{Monte Carlo Simulations}

For the first part of the work, Geant4 version 9.6 patch-02 (Agostinelli et al., 2003) was employed to simulate a 3.8 MeV mono-energetic proton pencil beam interacting with the micro-calorimeter. The positional information of individual energy transfers (called interactions in Geant4) and the amount of energy deposited in each layer was recorded for input into the heat transfer model. In addition, the model was used to produce the expected microdosimetric spectra of the incident particle. The micro-calorimeter model built for this investigation has a simple geometry constructed of three layers: the TE absorber, the SC absorber and the silicon substrate. The micro-calorimeter is connected to a printed circuit board (PCB) and mounted on a sample holder, both of which are not included in the model. Modelling the PCB and the sample holder are computationally intensive and have no effect on the thermal analysis of the micro-calorimeter since they can be assumed to be part of the constant temperature environment. The thickness of each layer and its mass are tabulated in table (1).

\begin{tabular}{l|c|c}
\hline \multicolumn{1}{c|}{ Layer } & Thickness $(\boldsymbol{\mu m})$ & Mass $(\mathbf{k g})$ \\
\hline TE absorber & 0.2 & $6.89 \times 10^{-14}$ \\
\hline SC absorber & 0.12 & $4.11 \times 10^{-13}$ \\
\hline Silicon Substrate & 360 & $7.55 \times 10^{-10}$ \\
\hline
\end{tabular}

Table 1: Components of the micro-calorimeter and the actual thicknesses used for simulations.

\section{Materials}

All the materials used for the construction of the micro-calorimeter are modelled in Geant4 using National Institute of Standards and Technology (NIST) material data. The G4NistManager class is used to derive the material from the NIST database. The materials are Carbon, Niobium and Silicon that are assigned to the TE absorber, the SC absorber and the silicon substrate respectively.

Physics

Two types of interactions are considered in this MC simulation. In Geant4 the interactions of photons and charged particles are governed by electromagnetic (EM) and hadronic physics. The main physics models used in this work for the EM processes are G4Livermore, which has been 


\section{ACCEPTED MANUSCRIPT}

validated at low energies, down to $100 \mathrm{eV}$ (Araujo et al., 2005), G4lonisation, G4PhotoElectricEffect, G4ComptonScattering, G4Bremsstrahlung and G4MultipleScattering (particle dependent). The models for hadronic processes include: G4HadronElasticProcess, G4InelasticProcess and G4BinaryLightlonReaction. The physics list employed in this model was previously validated by Galer (2012).

The production cut parameter in Geant4 allows the user to specify a threshold distance, which restricts secondary particles to be produced only if they have enough energy to travel this distance or further. To simulate and measure the track information in the thin film absorbers accurately, the minimum production cut of $0.1 \mu \mathrm{m}$, which is approximately equivalent to a $100 \mathrm{eV}$ threshold energy, was assigned to the absorbers. A different production cut of $2.5 \mu \mathrm{m}$ was defined for tracking particles in the silicon substrate. This is because the substrate thickness is approximately $1500 \mathrm{x}$ larger than that of both absorbers together. The potential for reducing the production cut in the silicon substrate was limited by the computation time for simulating the thermal relaxations.

Due to the stochastic nature of interactions no two particle tracks are the same, resulting in different responses of the micro-calorimeter. An energy deposition filter was determined by simulating 20 million proton particles to interact with the micro-calorimeter, to determine the most common particle tracks. The filter was based on the amount of energy deposited by the primary and secondary particles in the TE and SC absorbers. The track information (i.e. position and magnitude of energy transfer) per particle was recorded for implementation in the heat transfer model.

\section{Heat Transfer Modelling}

The particle track information obtained from the Geant4 MC simulation is communicated as input to the heat transfer model for thermal analysis of the micro-calorimeter. COMSOL Multiphysics, a finite element solver software package, was employed to build the heat transfer model with the latest version (5.2) being used for this work (COMSOL, 2015).

\section{Geometry}

The geometry built in COMSOL was modified to reduce the computation time. The accuracy that can be obtained from any finite element method model is directly related to the number of elements of the finite element mesh that is used. Preliminary heat transfer simulations on the micro-calorimeter with actual sizes, presented in table (1), confirmed that the computation time for a suitable meshing structure of such geometry were long and impractical if many tracks were to be simulated. The very thin film sizes of the TE absorber $(0.2 \mu \mathrm{m})$ and the SC absorber $(0.12 \mu \mathrm{m})$ on the $360 \mu \mathrm{m}$ thick silicon 


\section{ACCEPTED MANUSCRIPT}

substrate forced the meshing structure to have a very large number of meshing elements in the order of $1 \times 10^{6}$. This resulted in each run taking approximately 30 minutes on a high performance computer (12 2.30Ghz Intel cores) which was undesirably long when considering many track simulations. Therefore, an alternative method of thickness scaling was developed in building the model.

Rescaling the TE and the SC absorber thickness to a size comparable to that of the silicon substrate $(360 \mu \mathrm{m})$ improved the meshing structure and the computation time. The thickness of the TE and the SC absorbers were scaled to $100 \mu \mathrm{m}$ and $60 \mu \mathrm{m}$ respectively. To maintain the thermal relaxation behaviour of the micro-calorimeter similar to that of the actual size, the thermal conductivity and density of both absorbers were scaled accordingly. This reduced the simulation time by a factor of 10 , i.e., to less than 3 minutes per run.

An optimised meshing was created for the model that incorporated every interaction caused by the particle traversing the micro-calorimeter which could be as high as 100 interactions depending on the energy of the incoming particle.

A maximum meshing element size was determined for each layer from the conduction length presented in table (2). The volume-averaged temperature of each layer was calculated in $0.1 \mathrm{~ns}$ time steps. The conduction length scale of this time resolution was determined employing equation (3).

$$
l=6 \sqrt{\alpha t}
$$

Where,

$I$ is the conduction length scale

$t$ is the shortest time step required after heat source is switched off

$\alpha$ is the thermal diffusivity of the material, which can be interpreted as a measure of thermal inertia

\begin{tabular}{l|c|c|c}
\hline Layer & TE absorber & SC absorber & Silicon substrate \\
\hline $\begin{array}{l}\text { Maximum element } \\
\text { size }(\mu \mathrm{m})\end{array}$ & 6.0 & 30.0 & 52.0 \\
\hline
\end{tabular}

Table 2: Conduction length for each layer assuming a $0.1 \mathrm{~ns}$ resolution. 


\section{ACCEPTED MANUSCRIPT}

In addition, the meshing for point-heat-sources representing energy transfer points were created with a maximum element size of $0.5 \mu \mathrm{m}$. The positions of the point-heat-sources were changed for each particle track resulting in a unique meshing structure for individual particle tracks. The bespoke meshing structure created, allowed accurate analysis of the thermal behaviour starting from the

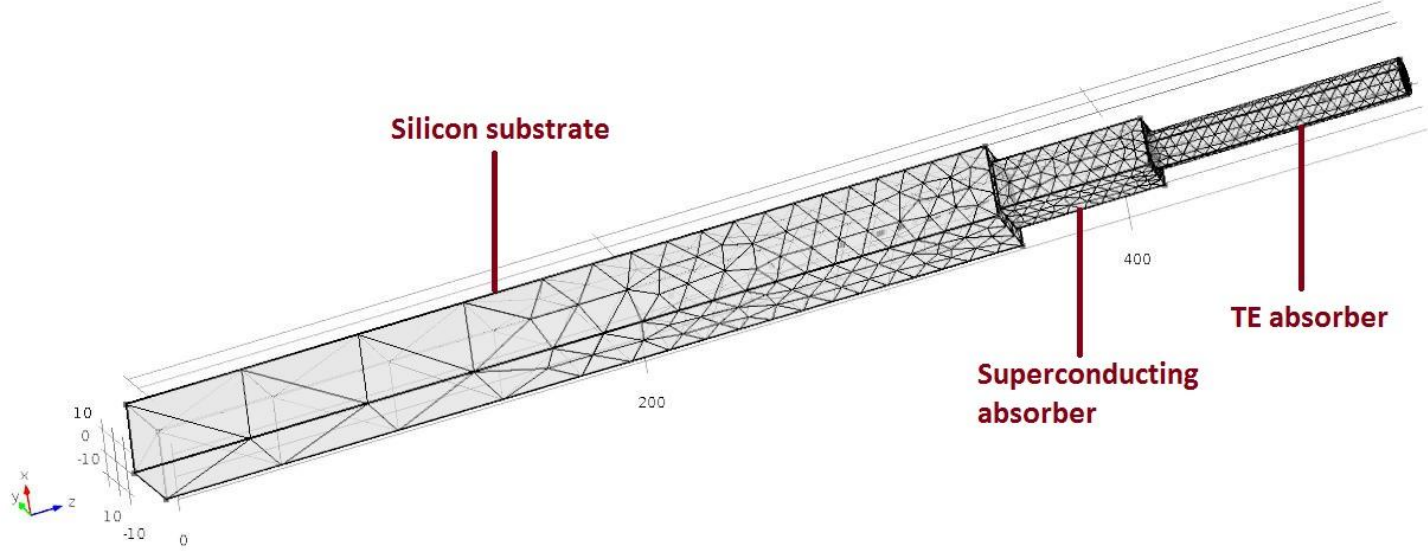

0

Figure 2: A typical meshing structure of the micro-calorimter model. The TE and the superconducting absorbers thicknesses have been scaled.

time that power is dissipated into the system as it conducts through the absorbers and into the silicon substrate. A typical meshing structure created when a $3.8 \mathrm{MeV}$ proton beam interacts with the micro-calorimeter is presented in figure (2).

A time control step function was defined in COMSOL for the insertion of power over 1 ns into the model which represents particle interactions.

\section{Material}

One of the most important factors influencing the behaviour of temperature in the COMSOL simulations is the thermal properties of the material used for modelling. The experiment is performed at cryogenic temperatures and the micro-calorimeter performance is optimised at $\approx 6 \mathrm{~K}$.

Measurements of the exact properties of the materials used in this investigation are not possible or require complex experiments that are out of scope of this study. The properties of materials closely representing the micro-calorimeter, reported in published data, were used for COMSOL simulations.

The Thermophysical Properties Research Center has published a comprehensive compilation of data that was used to select the thermal conductivity and specific heat capacity of $\mathrm{Si}$ and $\mathrm{Nb}$ (Touloukian et al., 1974, 1970).

The TE absorber used in the micro-calorimeter consists of amorphous carbon deposited by electron beam induced deposition of naphthalene gas. The thermal properties of the TE absorber depends on 


\section{ACCEPTED MANUSCRIPT}

the density of the amorphous carbon which is difficult to determine once deposited. Unlike the thermal properties of graphite, there is lack of published data on thermal properties of amorphous carbon film at the intended temperature $\approx 6 \mathrm{~K}$. The specific heat capacity of the amorphous carbon film at cryogenic temperatures was chosen from the only published data available by Takahashi and Westrum in 1970 (Takahashi and Westrum, 1970). The thermal conductivity of the amorphous carbon were taken from recommended data by Ho et al. in "Thermal conductivity of the elements: A comprehensive Review" (Ho et al., 1974).

\section{Determination of the correction factor}

The advantage of the computational model over experiment is that the model can treat the layers independently. This capability was utilised to develop a method to distinguish the temperature rise due to a) the heat conducted from the adjacent layers and b) the heat from direct energy deposition in each domain.

For every incident proton particle four different interaction conditions were built in the heat transfer model as shown in figure (3).

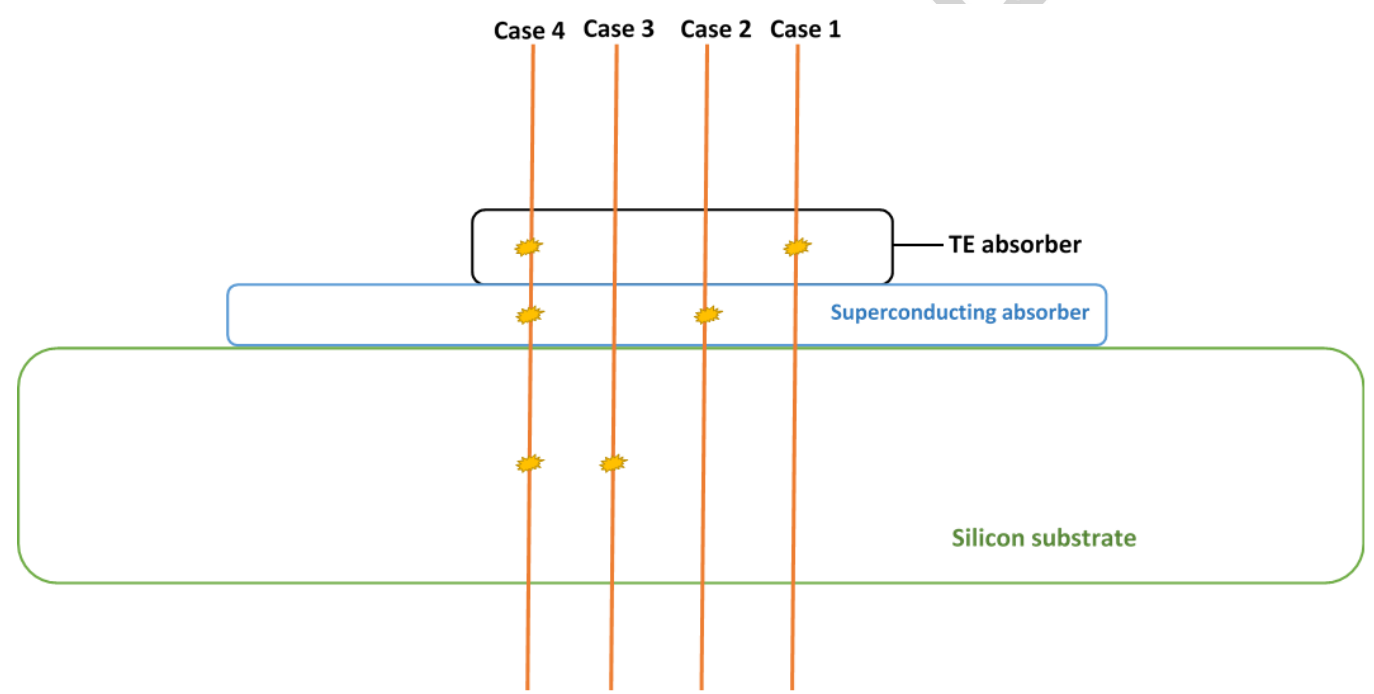

Figure 3: The 4 cases built for every incident proton to determine the temperature contributions. Viewing the geometry in zx planes.

Case 1, 2 and 3 represent the hypothetical conditions of energy deposition in the TE absorber, the SC absorber and the silicon substrate respectively. Case 4 represents the experimental condition where the incident proton interacts and deposits energy in every layer of the micro-calorimeter.

The volume-averaged temperature rise in each layer was recorded for all 4 cases over $10 \mathrm{~ns}$. A detectable signal is obtained from the micro-calorimeter when there is a temperature change in the SC absorber. In an ideal measurement situation, the incident proton would only deposit energy in the TE absorber (case 1) and the signal from the micro-calorimeter will solely be due to the heat 


\section{ACCEPTED MANUSCRIPT}

conducted from the TE absorber to the SC absorber. However, this was proven to be unlikely observing $M C$ simulations where the incident particle deposited energy in all layers of the microcalorimeter (case 4).

Utilising this method, the temperature rise due to direct energy deposition or heat contribution from adjacent layers can be determined. The average volume temperature rise of the SC absorber while simulating case 1 , represents temperature rise caused by heat conduction from the TE absorber. The temperature rise in the SC absorber, as a result of heat conducted from the silicon substrate, can be determined from simulating case 3 .

The sum of temperature rises in a layer while simulating case 1,2 and 3 should be equal to that of simulating case 4 . The difference between these calculations is an indication of the systematic uncertainty in the model.

The method developed is useful, detailed and powerful in determining possible corrections to the signal from the micro-calorimeter. However, it is necessary to simulate the effect of a large number of particles in order for the results to be statistically reliable. Simulating 4 different cases for every incident particle and manually inputting the parameters for every particle track is laborious and unfeasible, therefore, the process was automated.

The novelty of this work, in addition to the determination of appropriate correction factors, is the method of coupling MC simulations into a heat transfer model for thermal analysis, which can be adopted for any other thermal analysis work with ionising radiation. The practicality of this method is enhanced by the automation process which is described in this section. The automation process was possible with the LiveLink ${ }^{\text {TM }}$ for MATLAB module that connected COMSOL Multiphysics with Matlab scripting.

A script was written in Matlab which performed the pre-processing on every track for use in the heat transfer model. The script reads in the track information obtained from MC simulations, applies the individual energy transfer position and the amount of energy deposited to the COMSOL model. The outcome included the average volume-averaged temperature of each layer in all the 4 cases, which were recorded for post processing.

The ratio of the average volume temperature rise, measured at $0.2 \mathrm{~ns}$ after power input, in the SC absorber when simulating case 1 and case 4 is calculated for 1,000 proton tracks. The ratio determined is the percentage heat contribution from the TE absorber into the SC absorber. The stochastic behaviour of the particle interaction results in fluctuation of the factor determined. 


\section{ACCEPTED MANUSCRIPT}

Therefore, the correction factor is calculated as the mean value for the percentage heat contribution from the TE absorber into the SC absorber.

\section{Applying the correction factor}

The correction factor was applied to the expected energy frequency distribution in the microcalorimeter. The corrected distribution was converted to a microdosimetric spectrum, which was compared to an ideal case simulated employing Geant4 MC simulations. The expected energy frequency distribution is obtained by considering energy deposition in both the TE and the SC absorbers, whereas in the ideal case only the energy deposited in the TE absorber is considered.

\section{3- Results \& discussion}

The temperature increase in the SC absorber assuming energy deposition in the TE absorber is shown in figure (4). This range of temperature change is easily detectable with the micro-

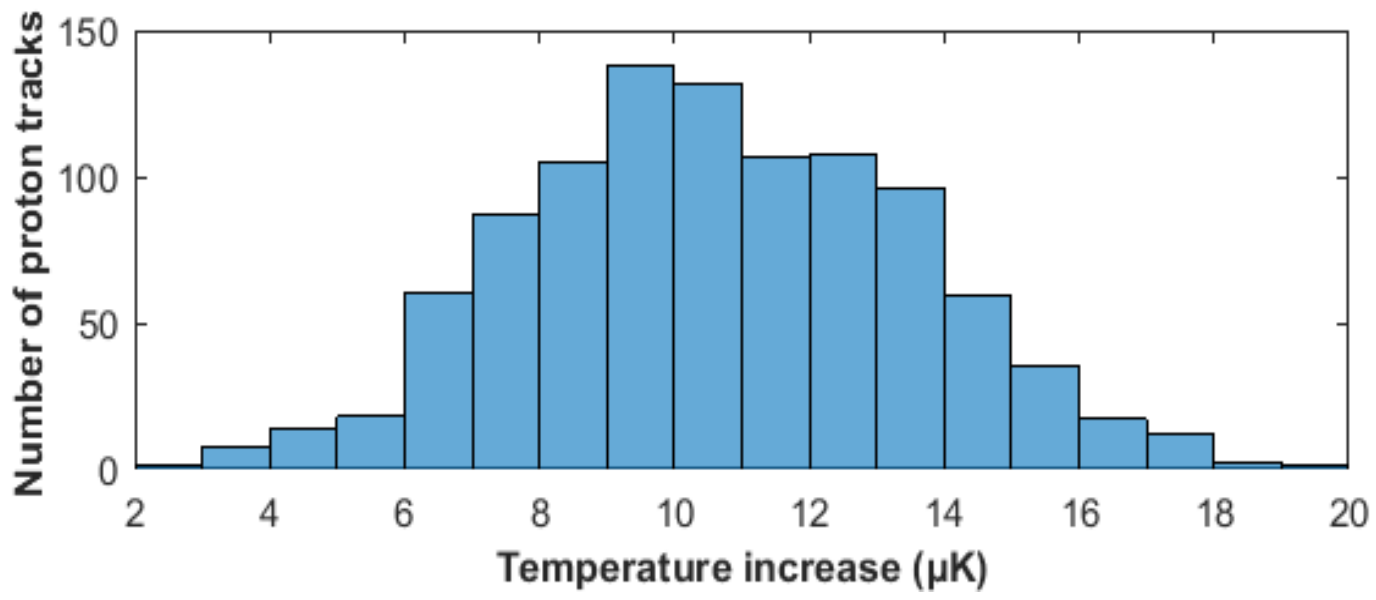

Figure 4: Histogram plot of temperature increase in the superconducting absorber caused by energy deposition in the TE absorber.

calorimeter.

The percentage heat contribution, per proton track, from the TE absorber resulting in a temperature change in the SC absorber is the correction factor required per track. The mean percentage heat contribution is determined indicating the mean correction factor required to correct the response of 


\section{ACCEPTED MANUSCRIPT}

the micro-calorimeter assuming most common tracks for a $3.8 \mathrm{MeV}$ proton pencil beam. The mean correction factor and the standard error of the mean was determined to exhibit a value of $50.15 \pm 0.02$. The response to every track is unique, hence there is a variation in the correction factors obtained for each individual proton track. The variation determined in terms of standard deviation has a value of $\sigma=28$.

A detailed analysis of the heat contributions show that the correction factor obtained is significantly influenced by the heat conducted from the silicon substrate to the SC absorber. At the relatively low initial beam energy of 3.8 MeV the protons fully stop in the silicon substrate depositing most of their energy in this layer. Therefore, a small change in the energy deposited in the silicon substrate has a significant effect on the determined correction factor. The effect is less pronounced in the case of energy deposition variation in TE and the SC absorbers.

The expected and ideal microdosimetric spectra using the micro-calorimeter was determined simulating 20 million proton particles with an energy of 3.8 MeV, shown in figure (5). The correction factor obtained is applied to the expected microdosimetric spectra simulated employing Geant4 MC simulations.

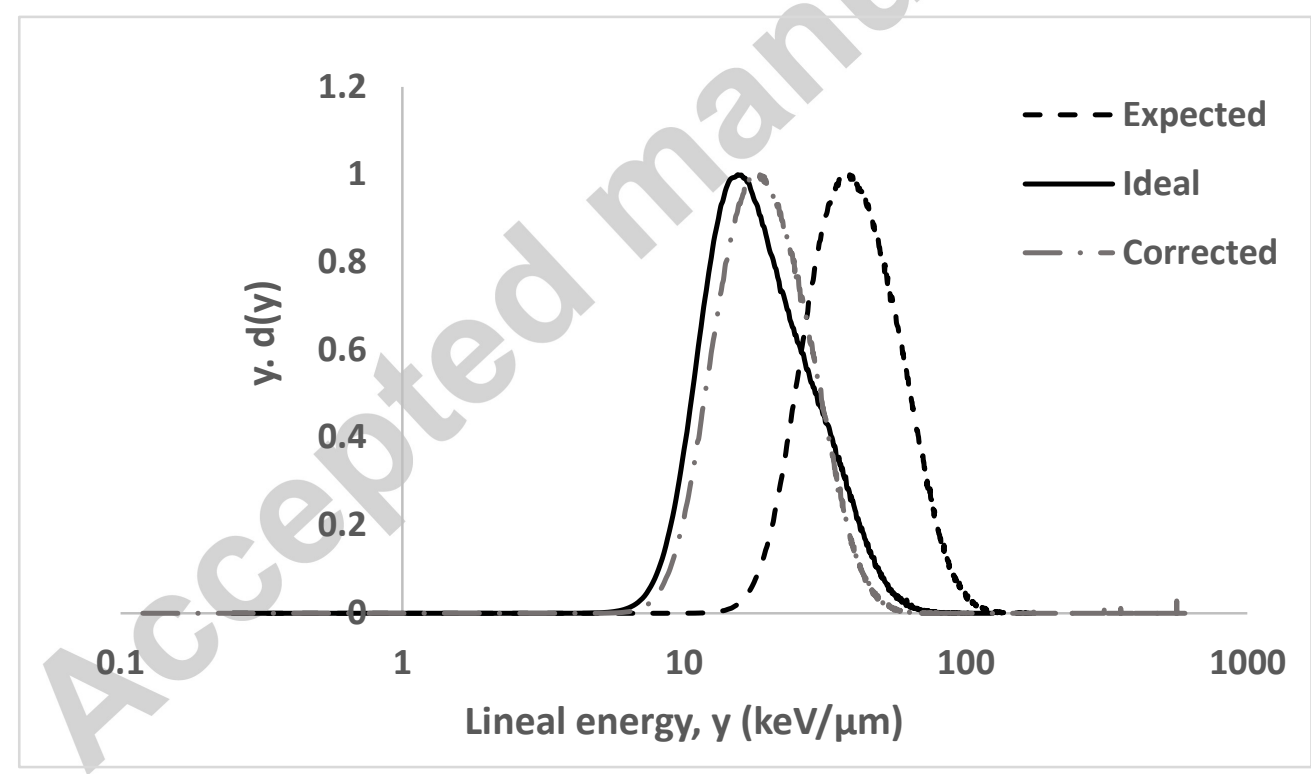

Figure 5: Expected, ideal and corrected microdosimetric spectra for a 3.8 MeV proton pencil beam.

The corrected spectrum has a good agreement with the hypothetical ideal spectrum, which is achieved if the particles deposit energy only in the TE absorber. This suggests that the method developed is feasible to correct the experimentally determined microdosimetric spectra. The large standard deviation on the mean correction factor of all the tracks could have caused the difference between the corrected and the ideal spectra. 


\section{ACCEPTED MANUSCRIPT}

\section{4- Conclusion}

A novel method was described that couples Monte Carlo simulations with a heat transfer model to determine the correction factor needed when measuring microdosimetric spectra of a $3.8 \mathrm{MeV}$ proton pencil beam with the micro-calorimeter. Automating the process allowed for thermal analysis of 1,000 proton tracks which can be increased the expense of longer computation time. The determined correction factor was applied to obtain a corrected microdosimetric factor which can then be used to determine a RBE value. The models are capable of simulating particles other than protons at higher energies.

\section{5- Future work}

The work is currently in progress to simulate particle beam energies higher than $3.8 \mathrm{MeV}$ and in the region of clinical proton beam energy. A single mean correction factor may not be applicable to a mixed particle beam hence an alternative method is being investigated, which would allow correction of mixed particle beams or wide spectrums. A redesign of the micro-calorimeter is also under investigation to eliminate the dominant effect of the silicon substrate on the correction factors. Furthermore, experimental work is in process to operate the micro-calorimeter at a microbeam facility for comparison with other microdosimetric detectors.

\section{Acknowledgment}

This work was supported by the Engineering and Physical Sciences Research Council (EPSRC) funding and the National Institute for Health Research (NIHR).

\section{References}

Agostinelli, S., Allison, J., Amako, K., Apostolakis, J., Araujo, H., Others, 2003. GEANT4 - A simulation toolkit. Nucl. Instruments Methods Phys. Res. Sect. A Accel. Spectrometers, Detect. Assoc. Equip. 506, 250-303. doi:10.1016/\$0168-9002(03)01368-8

Araujo, H., Howard, A., Chauvie, S., Guatelli, S., Mantero, B., Mascialino, M., Pia, M.G., Piergentili, M., Saliceti, S., Ivanchenko, V., Longo, F., 2005. Geant4 low energy electromagnetic physics, in: The Monte Carlo 2005 MC2005 Conference.

Brenner, D.J., Zaider, M., 1998. Estimating RBEs at clinical doses from microdosimetric spectra. Med. Phys. 25, 1055-1057. doi:https://doi.org/10.1118/1.598278

COMSOL, 2015. COMSOL Multiphysics reference manual. 


\section{ACCEPTED MANUSCRIPT}

Daşu, A., Toma-Daşu, I., 2008. What is the Clinically Relevant Relative Biologic Effectiveness? A Warning for Fractionated Treatments With High Linear Energy Transfer Radiation. Int. J. Radiat. Oncol. Biol. Phys. 70, 867-874. doi:10.1016/j.ijrobp.2007.07.2358

De Nardo, L., Cesari, V., Dona, G., Magrin, G., Colautti, P., Tornielli, G., 2004. mini-TEPC for radiation therapy. Radiat. Prot. Dosimetry 108, 345-352. doi:https://doi.org/10.1093/rpd/nch023

Galer, S., 2012. Development of a Microbolometer for Microdosimetry of lonising Radiation. University of Surrey.

Galer, S., Hao, L., Gallop, J., Palmans, H., Kirkby, K., Nisbet, A., 2011. Design concept for a novel SQUID-based microdosemeter. Radiat. Prot. Dosimetry 143, 427-431. doi:10.1093/rpd/ncq475

Hao, L., Gallop, J.C., Gardiner, C., Josephs-Franks, P., Macfarlane, J.C., Lam, S.K.H., Foley, C., 2003. Inductive superconducting transition-edge detector for single-photon and macro-molecule detection. Supercond. Sci. Technol. 16, 1479-1482. doi:10.1088/0953-2048/16/12/035

Ho, C., Powell, R., Liley, P., 1974. Thermal conductivity of elemetns: a comprehensive review.

Jones, B., Dale, R.G., 2000. Estimation of optimum dose per fraction for high LET radiations: Implications for proton radiotherapy. Int. J. Radiat. Oncol. Biol. Phys. 48, 1549-1557. doi:10.1016/S0360-3016(00)00781-1

Kellerer, A.M., 1985. Fundamentals of Microdosimetry, in: THE DOSIMETRY OF IONIZING RADIATION. pp. 77-162.

Kellerer, A.M., 1984. A SURVEY OF MICRODOSIMETRIC A.M. KELLERER Instrtut fiir 14, 169-172.

Matsuura, T., Egashira, Y., Nishio, T., Natsumoto, Y., Kohno, R., Ogino, T., 2010. Apparent absence of a proton beam dose rate effect and possible differences in RBE between Bragg peak and plateau. Med Phys 37, 5376-5381. doi:10.1118/1.3490086

Microdosimetry ICRU Report 36, 1983.

Moro, D., Colautti, P., Gualdrini, G., Masi, M., Cote, V., De Nardo, L., Tornielli, G., 2006. Two miniaturised TEPCs in a single detector for BNCT microdosimetry. Radiat. Prot. Dosimetry 122, 396-400. doi:https://doi.org/10.1093/rpd/ncl484

Paganetti, H., Niemierko, A., Ancukiewicz, M., Gerweck, L.E., Goitein, M., Loeffler, J.S., Suit, H.D., 2002. Relative biological effectiveness (RBE) values for proton beam therapy. Int. J. Radiat. Oncol. 53, 407-421. doi:10.1016/S0360-3016(02)02754-2 


\section{ACCEPTED MANUSCRIPT}

Particle Therapy Co-Operative Group, 2016. Particle Therapy Co-Operative Group [WWW

Document]. URL http://www.ptcog.ch/index.php/facilities-in-operation (accessed 7.27.16).

Takahashi, Y., Westrum, E.F., 1970. Glassy Carbon low-temperature thermodynamic properties. J.

Chem. Thermodyn. 2, 847-854. doi:10.1016/0021-9614(70)90028-5

Tilly, N., Grusell, E., Kimstrand, P., Lorin, S., Gajewski, K., Pettersson, M., Bäcklund, A., Glimelius, B., 2007. Development and verification of the pulsed scanned proton beam at The Svedberg Laboratory in Uppsala. Phys. Med. Biol. 52, 2741-2754. doi:10.1088/0031-9155/52/10/008

Touloukian, Y., Powell, R., Ho, C., Klemens, P., 1970. Thermal Conductivity - Metallic Elements and Alloys, in: Thermophysical Properties of Matter- The TPRC Data Series Volume 1. pp. 246-256.

Touloukian, Y., Powell, R., Ho, C., Nicolaou, M., 1974. Specif Heat-Metallic Elements and Alloys, in: Thermophysical Properties of Matter- The TPRC Data Series Volume 4. pp. 204-210.

Highlights

- Thermal analysis of a novel micro-calorimeter for use in particle therapy

- Automated process of coupling Monte Carlo simulations with finite element analysis

- Determining a correction factor for a 3.8 MeV micro-beam 